\title{
Phase separation of incompressible binary fluids with Lattice Boltzmann Methods
}

\author{
Aiguo $\mathrm{Xu}^{1}, \mathrm{G}$. Gonnella ${ }^{1,2}$ and A. Lamura ${ }^{3}$ \\ ${ }^{1}$ Istituto Nazionale per la Fisica della Materia, Unità di Bari, and Dipartimento di Fisica, \\ Università di Bari, and TIRES, Center of Innovative Technologies for Signal Detection and \\ Processing, via Amendola 173, 70126 Bari, Italy \\ ${ }^{2}$ INFN, Sezione di Bari, via Amendola 173, 70126 Bari \\ 3 Istituto Applicazioni Calcolo, CNR, Sezione di Bari, \\ Via Amendola 122/I, 70126 Bari, Italy
}

(November 8, 2018)

\begin{abstract}
We introduce new versions of lattice Boltzmann methods (LBM) for incompressible binary mixtures where fluctuations of total density are inhibited. As a test for the improved algorithms we consider the problem of phase separation of two-dimensional binary mixtures quenched from a disordered state into the coexistence region. We find that the stability properties of LBM are greatly improved. The control of density fluctuations and the possibility of running longer simulations allow a more precise evaluation of the growth exponent.
\end{abstract}

KEY WORDS: Lattice Boltzmann methods; binary mixtures; phase separation. 


\section{INTRODUCTION}

Lattice Boltzmann methods (LBM) are computational schemes for solving the macroscopic equations of fluid systems $[1,2]$. In the case of binary mixtures they have been largely used to study the dynamics of systems described by the Navier-Stokes and convectiondiffusion equations [3]. The advantages of LBM compared with standard methods for partial differential equations are particularly relevant in problems involving complex boundaries such as multiphase flow in porous media $[4,1,2]$. The large number of applications of LBM in studies on complex fluids [5-7], polymer mixtures [8], liquid crystals [9], etc. demonstrates the versatility of the method. In studies of spinodal decomposition of binary mixtures a version of LBM based on a free-energy approach, where the equilibrium distributions can be consistently chosen with the thermodynamics of the system, has been largely used [10-12].

The stability properties of LBM are always crucial, especially in phase separation studies where long lasting simulations are needed to establish the growth properties. Indeed, the average size of domains of the two phases generally grows with power law behavior and one wants to determine the exponent $[13,14]$. However, previous studies showed the existence of a stability problem: total density fluctuations can suddenly grow in an uncontrolled way interrupting simulations and making problematic the determination of the growth exponent $[12]$.

In this paper we consider new versions of LBM where the stability of the algorithm is improved. We have checked the stability properties by studying the problem of phase separation of two-dimensional binary mixtures. This is a severe test for stability since density fluctuations are enhanced by the presence of many interfaces in the system though they should be negligible in incompressible fluids. We find that, with the new algorithms, density fluctuations are largely suppressed and a more reliable determination of the growth exponent can be obtained.

The incompressible Navier-Stokes equation can be obtained from lattice Boltzmann equations if density fluctuations are negligible [1]. Compressibility effects, intrinsic to LBM, can produce some serious errors in numerical simulations of incompressible fluids. Due to these reasons improved versions of LBM have been introduced for the case of a simple fluid $[15,16]$. New schemes are based on the explicit elimination in the equilibrium distributions of higher order terms in the Mach number [17] or on the introduction of a feedback mechanism [18]. With these improvements it has been shown that analytical solutions known for particular flows are better reproduced than with old LBM versions.

The above methods are here developed and introduced for the first time in the case of binary mixtures. The algorithms are presented in Section II. In Section III we study the problem of phase separation comparing the results obtained with different LBM versions. The role of boundary conditions on the problem of stability is examined in Section IV. Finally, in Section V, we discuss our results and draw our conclusions.

\section{THE MODEL}

Our simulations are based on the free-energy approach developed by Orlandini et al. [10] and Swift et al. [11]. In this scheme a suitable free energy is introduced to control the equilibrium properties of the system. 


\section{A. The lattice Boltzmann scheme}

The free-energy functional used in the present study is

$$
\mathcal{F}=\int d \mathbf{r}\left[\frac{1}{3} n \ln n+\frac{a}{2} \varphi^{2}+\frac{b}{4} \varphi^{4}+\frac{\kappa}{2}(\nabla \varphi)^{2}\right]
$$

where $n$ is the total density and $\varphi$ is the density difference between the two fluids. The term in $n$ gives rise to a positive background pressure. The terms in $\varphi$ in the free-energy density $f(n, \varphi)$ correspond to the usual Ginzburg-Landau expression typically used in coarse-grained models of phase separation [14]. The polynomial terms relate to the bulk properties of the fluid. While the parameter $b$ is always positive, $a$ allows to distinguish between a disordered $(a>0)$ and a segregated mixture $(a<0)$ where two pure phases with $\varphi= \pm \sqrt{-a / b}$ coexist. We will consider quenches into the coexistence region with $a<0$ and $b=-a$ so that the equilibrium values for the order parameter are $\varphi= \pm 1$. The gradient term is related to the surface tension. The other thermodynamic properties of the fluid follow from the free energy (1). The chemical potential difference between the two fluids is given by $\Delta \mu=\delta \mathcal{F} / \delta \varphi=a \varphi+b \varphi^{3}-\kappa \nabla^{2} \varphi$. The thermodynamic pressure tensor $P_{\alpha \beta}^{t h}$ is given by

$$
\begin{aligned}
P_{\alpha \beta}^{t h} & =\left(n \frac{\delta \mathcal{F}}{\delta n}+\varphi \frac{\delta \mathcal{F}}{\delta \varphi}-f(n, \varphi)\right) \delta_{\alpha \beta}+\kappa \partial_{\alpha} \varphi \partial_{\beta} \varphi \\
& =\frac{1}{3} n \delta_{\alpha \beta}+P_{\alpha \beta}^{c h e m}
\end{aligned}
$$

where the chemical part is $P_{\alpha \beta}^{c h e m}=\left(\frac{a}{2} \varphi^{2}+\frac{3}{4} b \varphi^{4}-\kappa \varphi\left(\nabla^{2} \varphi\right)-\frac{\kappa}{2}(\nabla \varphi)^{2}\right) \delta_{\alpha \beta}+\kappa \partial_{\alpha} \varphi \partial_{\beta} \varphi$.

The lattice Boltzmann model is defined on the two-dimensional square lattice with the following nine velocity vectors: $\mathbf{e}_{0}=(0,0), \mathbf{e}_{i}=(\cos [(i-1) \pi / 2], \sin [(i-1) \pi / 2]) c, i=$ $1,2,3,4, \mathbf{e}_{i}=(\cos [(i-5) \pi / 2+\pi / 4], \sin [(i-5) \pi / 2+\pi / 4]) \sqrt{2} c, i=5,6,7,8$, where $c=$ $\Delta x / \Delta t$, and $\Delta x$ and $\Delta t$ are the lattice constant and the time step, respectively. Two sets of distribution functions $f_{i}(\mathbf{r}, t)$ and $g_{i}(\mathbf{r}, t)$ evolve according to a single relaxation-time Boltzmann equation that is discrete in both time and space [19,20]:

$$
\begin{aligned}
& f_{i}\left(\mathbf{r}+\mathbf{e}_{i} \Delta t, t+\Delta t\right)-f_{i}(\mathbf{r}, t)=-\frac{1}{\tau}\left[f_{i}(\mathbf{r}, t)-f_{i}^{e q}(\mathbf{r}, t)\right] \\
& g_{i}\left(\mathbf{r}+\mathbf{e}_{i} \Delta t, t+\Delta t\right)-g_{i}(\mathbf{r}, t)=-\frac{1}{\tau_{\varphi}}\left[g_{i}(\mathbf{r}, t)-g_{i}^{e q}(\mathbf{r}, t)\right]
\end{aligned}
$$

where $\tau$ and $\tau_{\varphi}$ are independent relaxation parameters. The distribution functions are related to the total density $n$, to the fluid momentum $n \mathbf{v}$ and to the density difference $\varphi$ through

$$
n=\sum_{i} f_{i}, \quad n \mathbf{v}=\sum_{i} f_{i} \mathbf{e}_{i}, \quad \varphi=\sum_{i} g_{i}
$$

These quantities are locally conserved in any collision process and, therefore, we require that the local equilibrium distribution functions $f_{i}^{e q}(\mathbf{r}, t)$ and $g_{i}^{e q}(\mathbf{r}, t)$ fulfill the Eqs. (5). The higher momenta of the local equilibrium distribution functions are defined so that the continuum equations pertinent to a binary fluid mixture can be obtained $[10,11]$ 


$$
\begin{gathered}
\sum_{i} f_{i}^{e q} e_{i \alpha} e_{i \beta}=c^{2} P_{\alpha \beta}^{t h}+n v_{\alpha} v_{\beta}, \\
\sum_{i} g_{i}^{e q} e_{i \alpha}=\varphi v_{\alpha}, \\
\sum_{i} g_{i}^{e q} e_{i \alpha} e_{i \beta}=c^{2} \Gamma \Delta \mu \delta_{\alpha \beta}+\varphi v_{\alpha} v_{\beta} .
\end{gathered}
$$

where $\Gamma$ is a coefficient related to the mobility of the fluid. The local equilibrium distribution functions can be expressed as an expansion at the second order in the velocity $\mathbf{v}[10,11]$.

$$
\begin{aligned}
& f_{0}^{e q}=A_{0}+C_{0} v^{2} \\
& f_{i}^{e q}=A_{I}+B_{I} v_{\alpha} e_{i \alpha}+C_{I} v^{2}+D_{I} v_{\alpha} v_{\beta} e_{i \alpha} e_{i \beta}+G_{I, \alpha \beta} e_{i \alpha} e_{i \beta} \quad i=1,2,3,4 \\
& f_{i}^{e q}=A_{I I}+B_{I I} v_{\alpha} e_{i \alpha}+C_{I I} v^{2}+D_{I I} v_{\alpha} v_{\beta} e_{i \alpha} e_{i \beta}+G_{I I, \alpha \beta} e_{i \alpha} e_{i \beta} \quad i=5,6,7,8
\end{aligned}
$$

and similarly for the $g_{i}^{e q}, i=0, \ldots, 8$. The coefficients in the previous expansions are explicitly written for convenience to compare the original scheme with the alternative ones introduced in the following subsections. A suitable choice is [21]

$$
\begin{gathered}
A_{0}=\frac{4}{9} n-\frac{5}{6} P_{\alpha \beta}^{c h e m} \delta_{\alpha \beta}, \quad A_{I}=\frac{1}{9} n+\frac{1}{6} P_{\alpha \beta}^{c h e m} \delta_{\alpha \beta}, \quad A_{I I}=\frac{A_{I}}{4} \\
B_{I}=\frac{n}{3 c^{2}}, \quad B_{I I}=\frac{B_{I}}{4} \\
C_{0}=-\frac{2 n}{3 c^{2}}, \quad C_{I}=-\frac{n}{6 c^{2}}, \quad C_{I I}=\frac{C_{I}}{4} \\
D_{I}=\frac{n}{2 c^{4}}, \quad D_{I I}=\frac{D_{I}}{4} \\
G_{I, \alpha \beta}=\frac{P_{\alpha \beta}^{t h}-\frac{1}{2} P_{\sigma \sigma}^{t h} \delta_{\alpha \beta}}{2 c^{2}}, \quad G_{I I, \alpha \beta}=\frac{G_{I, \alpha \beta}}{4}
\end{gathered}
$$

The expansion coefficients for the $g_{i}^{e q}$ can be obtained from the previous ones with the formal substitutions $n \rightarrow \varphi$ and $P_{\alpha \beta}^{t h} \rightarrow \Gamma \Delta \mu \delta_{\alpha \beta}$. The quantities $P_{\alpha \beta}^{t h}$ and $\Delta \mu$, which appear in the coefficients of the equilibrium distribution functions, can be calculated from (1).

It has been shown in Refs. [10,11], using a Chapman-Enskog expansion [22], that the above described lattice Boltzmann scheme simulates at second order in $\Delta t$ the continuity equation

$$
\partial_{t} n+\partial_{\alpha}\left(n v_{\alpha}\right)=0+o\left(\Delta t^{2}\right),
$$

and the Navier-Stokes equation 


$$
\begin{aligned}
& \partial_{t}\left(n v_{\beta}\right)+\partial_{\alpha}\left(n v_{\alpha} v_{\beta}\right)=-\partial_{\beta} p-\partial_{\alpha} c^{2} P_{\alpha \beta}^{c h e m}+\nu \partial_{\gamma}\left(n\left(\partial_{\beta} v_{\gamma}+\partial_{\gamma} v_{\beta}\right)\right)+ \\
& +3 \nu \partial_{\alpha}\left(\partial_{t} P_{\alpha \beta}^{c h e m}-\partial_{\gamma}\left(n v_{\alpha} v_{\beta} v_{\gamma}\right)-\left(v_{\beta} \partial_{\gamma} P_{\alpha \gamma}^{c h e m}+v_{\alpha} \partial_{\gamma} P_{\beta \gamma}^{c h e m}\right)\right)+o\left(\Delta t^{2}\right)
\end{aligned}
$$

where the isotropic pressure contribution is

$$
p=c_{s}^{2} n,
$$

$c_{s}=c / \sqrt{3}$ and $\nu=(\tau-1 / 2) c_{s}^{2} \Delta t$ being the speed of sound and the kinematic viscosity, respectively. The first line of Eq. (16) corresponds to the standard Navier-Stokes equation. The second line contains spurious terms. Nonetheless, these terms, being gradient terms with higher order derivatives, are negligible compared to the ones in the first line when all hydrodynamic fields vary smoothly on the lattice scale $[11,12]$. Moreover, a convectiondiffusion equation is also recovered:

$$
\begin{aligned}
\partial_{t} \varphi+\partial_{\alpha}\left(\varphi v_{\alpha}\right)= & \Theta \nabla^{2} \Delta \mu+ \\
& -\left(\tau_{\varphi}-\frac{1}{2}\right) \Delta t \partial_{\alpha}\left(\frac{\varphi}{n} \partial_{\alpha} p+\frac{\varphi}{n} \partial_{\beta} c^{2} P_{\alpha \beta}^{c h e m}\right)+o\left(\Delta t^{2}\right)
\end{aligned}
$$

where the macroscopic mobility is $\Theta=\Gamma\left(\tau_{\varphi}-1 / 2\right) c^{2} \Delta t$. As before, the spurious terms in the second line of Eq. (18) are also expected to be small.

If the density fluctuations are negligible, the continuity and Navier-Stokes equations (15)-(16) describe the behavior of an incompressible fluid. However, this is not always the case in LBM where the compressibility effects can produce errors in numerical simulations. In the next two subsections we will modify the described LBM in order to reduce unphysical compressibility effects.

\section{B. The limit of small Mach number}

The first scheme that we consider and adapt to our LBM is the one introduced in Ref. [17]. Previous studies for incompressible fluids have shown that the density fluctuations, $\delta n$, are expected to be of order $o\left(M^{2}\right)$ in the limit $M \rightarrow 0, M=v / c_{s}$ being the Mach number $[15,23]$. We enforce this condition in the method. By substituting $n=n_{0}+\delta n$ into the equilibrium distribution functions (9), $n_{0}$ being the specified constant value of density, and neglecting the terms proportional to $\delta n(\mathbf{v} / c)$, and $\delta n(\mathbf{v} / c)^{2}$, which are of the order $o\left(M^{3}\right)$ or higher, we find that the net effect is to replace $n$ with $n_{0}$ into the expansion coefficients (11)-(13) keeping unchanged the rest of the scheme. In this way the terms in the equilibrium distribution functions (9) are of the order $o\left(M^{2}\right)$ or lower. The condition $M \ll 1$ is satisfied in our runs where it is always $M<0.01$.

In this case the Chapman-Enskog expansion gives the continuity equation

$$
\partial_{\alpha} v_{\alpha}=0+o\left(\Delta t^{2}\right)+o\left(M^{2}\right)
$$

the incompressible Navier-Stokes equation

$$
\partial_{t} v_{\beta}+v_{\alpha} \partial_{\alpha} v_{\beta}=-\frac{1}{n_{0}} \partial_{\beta} p-\frac{1}{n_{0}} \partial_{\alpha} c^{2} P_{\alpha \beta}^{c h e m}+\nu \nabla^{2} v_{\beta}+o\left(\Delta t^{2}\right)+o\left(M^{3}\right),
$$

and the convection-diffusion equation

$$
\partial_{t} \varphi+\partial_{\alpha}\left(\varphi v_{\alpha}\right)=\Theta \nabla^{2} \Delta \mu+o\left(\Delta t^{2}\right)+o\left(M^{3}\right),
$$

where we have not written again the spurious terms of Eqs. (16)-(18). 


\section{A feedback mechanism}

In Ref. [18] a different algorithm based on a feedback mechanism is proposed to keep the density nearly constant in LBM for a single fluid. Here we adapt this scheme to our model combining it with the one previously described. We use for the expansion coefficients (10) the following form:

$$
\begin{aligned}
A_{0} & =a_{0} n-\frac{5}{6} P_{\alpha \beta}^{c h e m} \delta_{\alpha \beta} \\
A_{I} & =a_{I} n+\frac{1}{6} P_{\alpha \beta}^{c h e m} \delta_{\alpha \beta} \\
A_{I I} & =\frac{A_{I}}{4}
\end{aligned}
$$

Let us note that these expressions coincide with (10) when $a_{0}=4 / 9$ and $a_{I}=1 / 9$. Here we only ask that $a_{0}+5 a_{I}=1$ to guarantee the total density conservation. We consider at each node and at each time step before collision the density $n$ fixing the value of $a_{I}$ as

$$
a_{I}=a_{I}^{0}-b\left(1-\frac{n}{n_{0}}\right)
$$

where $a_{I}^{0}=1 / 9$ and $b$ is a positive constant given by experience. In this paper we generally use $b=0.01$. The value of $b$ is flexible, but if it is too large the simulation will be unstable. The value of $a_{0}$ follows from the relation between $a_{0}$ and $a_{I}$. These values are then used in (22). This choice forces, in the next streaming step, more (less) particles to leave the node with $n>n_{0}\left(n<n_{0}\right)$, resulting in $n \rightarrow n_{0}$.

By using a Chapman-Enskog expansion and neglecting terms of order $o\left(M^{3}\right)$ or higher we find the continuity equation (19), the incompressible Navier-Stokes equation (20) where now the isotropic pressure contribution is

$$
p^{\prime}=3 a_{I} c^{2} n=c_{s}^{2} n-3 c^{2} b\left(n_{0}-n\right)+o\left(\delta n^{2}\right)
$$

and the convection-diffusion equation (21). For the convenience of description, we label the

old scheme as A, the scheme described in subsection B as B, the scheme combining scheme $\mathrm{B}$ and the feedback mechanism as $\mathrm{C}$.

\section{PHASE SEPARATION WITH PERIODIC BOUNDARY CONDITIONS}

After a binary mixture is quenched into the coexistence region and domains of the two phases are well established, the typical size of domains generally grows as $R(t) \sim t^{\alpha}$. A simple scaling analysis of the Navier-Stokes and of the convection-diffusion equations shows that three regimes can be found depending on the role played by hydrodynamic degrees of freedom. At high viscosity the domain growth is governed by a diffusive mechanism and $\alpha=1 / 3$ [24]. When hydrodynamics becomes relevant, the laws $R(t) \sim t$ or $R(t) \sim t^{2 / 3}$ are expected depending on whether viscous forces or inertial effects dominate, respectively $[13,14]$. However, in real systems the situation is more complex. The physical mechanism responsible for viscous growth is not operating in the two-dimensional case [25] and, indeed, 
this regime has never been observed in simulations [3]. In this paper, since we address the question of stability which becomes more critical at low viscosities, we will focus on cases corresponding to the inertial regime.

The average size of domains $R(t)$ can be calculated as the first momentum of the structure factor, that is

$$
R(t)=\frac{\int d \vec{k} C(\vec{k}, t)}{\int d \vec{k}|k| C(\vec{k}, t)}
$$

where

$$
C(\vec{k}, t)=\langle\varphi(\vec{k}, t) \varphi(-\vec{k}, t)\rangle
$$

and $\langle\cdot\rangle$ is the average over different histories.

In Fig. 1 we show the behavior of $R(t)$ for the sets of parameters listed in Table I. Similar behaviors have been also observed in many other cases here not reported. We have also not reported the worst situations occurring at very low $\tau$, when the scheme A gets very soon unstable $(t \simeq 100)$ and the new schemes are not significantly better. All the cases of Table I refer to situations where phase separation has already generated well-formed macroscopic domains and a significant evolution of $R(t)$ can be observed. For each set, results obtained with the schemes A, B and C are compared. While the scheme A can be used only before density fluctuations start to grow indefinitely, the simulations with the new schemes remain stable at all times for most of the cases considered. We have found only few cases (e.g., set 6 at the lowest considered viscosity in Table I) where the schemes B and C are affected by instability; however, the instability with the scheme $\mathrm{C}$ occurs when the system is almost completely phase separated, later than with the scheme B and much later than with the scheme A.

All the cases shown correspond to the inertial regime and for reference we have plotted the line with slope $2 / 3$. The sets 1 and 2 only differ in the value of the mobility $\Gamma$. The main difference is that, before reaching the hydrodynamic $2 / 3$ regime, the growth is faster when the mobility is higher as it can be observed in Fig. 1. The slowing down of growth at large times is due to finite size effects appearing when the size of domains becomes comparable with the lattice size.

In order to understand the effects of density fluctuations, we show in Fig. 2 pictures of the system for the parameter set 2 at the time $t=5895$ when the scheme A becomes unstable. Simulations obtained with the old and the new schemes can be compared using the same initial conditions. Differences in the order parameter field can be observed due to the different treatment of density fluctuations. When the scheme A is used the density plot shows the appearing of a peak which will grow indefinitely. The presence of the instability can be also observed in the middle low region of the concentration field as a white stripe. In the case with the scheme B fluctuations remain limited around the average value $n=1$ and are larger in correspondence of interfaces. The velocity field also shows an instability with the scheme A while with the new scheme regular local flows can be observed.

¿From sets 3 and 6 of Fig. 1 we observe that $R(t)$ behaves more regularly when calculated with the scheme C. This allows a better determination of the growth exponent. The effects of using a bigger lattice size can be seen in Fig. 1 for cases 4 and 5 . 
In recent simulations of three-dimensional systems based on the original algorithm the growth exponent was obtained by fitting the results of $R(t)$ with the function $f(t)=c(t-d)^{\alpha}$ in a time interval from the inflexion point to the onset of the instability [12]. This procedure, as admitted by the same authors of Ref. [12] and discussed also in Ref. [26], is very sensitive to small variations of the initial time considered, so that the evaluation of the growth exponent is problematic. We have applied a similar procedure to our results confirming the sensitivity to small variations of the initial time considered and finding different values for the exponent when the old or the new more stable schemes are applied. For example, for the set 2 with the old method, we find $\alpha=0.58$ if we start from the inflexion point located at $t=3000$ and $\alpha=0.61$ if data are fitted from $t=4000$ (the run becomes unstable at $t=5985$ ). If we use the longer series of data obtained with the scheme $\mathrm{C}$ we get $\alpha=0.64$ starting from the inflexion point or at a later time. For the set 4 the fitting procedure for the scheme A gives values of $\alpha$ in the range $0.50-0.55$ changing the initial time for the fitting procedure, while the scheme $\mathrm{B}$ gives values in the range $\alpha=0.64-0.67$. Other cases are also worst because sometimes, like for the set 3 , a reliable determination of the exponent is not possible with the old scheme.

\section{STABILITY WITH WALLS}

In this Section we discuss how the presence of boundary conditions with walls affects the stability of simulations of phase separation. Boundary conditions with walls are present in problems where a flow is applied to the system, e. g. shear flow, or in situations with geometrically complex boundaries.

Fluctuations of density are generally larger close to the walls due to the fact that the propagation and collision steps involve a reflection on the boundary. Then, if the density is larger than the average value in a point close to a wall, the reflection tends to enhance this fluctuation. An example of the behavior of density just before the system becomes unstable is given in Fig. 3 which shows clearly that the instability appears close to the walls.

Also for the case with walls we have applied the schemes described in Sec. II for controlling the density fluctuations. In all the considered cases instabilities in simulations with walls occur much before than in simulations with periodic boundary conditions. In Table II we have reported the stability results for the original and the new scheme $\mathrm{C}$. The comparison shows that in most of the cases the new algorithm can be very useful for running longer simulations of phase separation.

\section{CONCLUSION}

In this paper we have considered two-dimensional Lattice Boltzmann methods for incompressible binary mixtures based on a free-energy functional approach. We have introduced mechanisms for controlling the total density fluctuations. These mechanisms were already shown useful for reproducing more accurately examples of analytical solutions for simple fluid flows.

In the case of binary mixtures, density fluctuations can induce numerical instabilities that make impossible to run long enough simulations as it is needed in problems like spinodal 
decomposition. We have used the new algorithms to study the phase separation of binary mixtures in the inertial regime at low viscosities when the effects of density fluctuations are worst. We have found that the stability of the simulations can be largely improved. For most of the parameters considered, differently from what happens with the original algorithm, it is possible to take under control density fluctuations eliminating the occurring of instabilities. This allows to study the properties of phase separating binary mixtures more reliably; in particular, a more accurate evaluation of the growth exponent can be given. As a future

research in this direction, it would be interesting to apply the LBM with improved stability to the case of a three-dimensional mixture.

\section{ACKNOWLEDGMENTS}

A.L. acknowledges INFM for partial support. 


\section{REFERENCES}

[1] S. Chen and G. D. Doolen, Annu. Rev. Fluid Mech. 30, 329 (1998).

[2] S. Succi, The Lattice Boltzmann Equation (Oxford University Press, New York, 2001).

[3] J. M. Yeomans, Ann. Rev. Comp. Physics, VII (2000).

[4] J. Olson and D. Rothman, J. Fluid. Mech. 341, 343 (1997).

[5] G. Gonnella, E. Orlandini, and J. M. Yeomans, Phys. Rev. Lett. 78, 1695 (1997).

[6] O. Theissen, G. Gompper, and D. M. Kroll, Europhys. Lett. 42, 419 (1998).

[7] A. Lamura, G. Gonnella, and J. M. Yeomans, Europhys. Lett. 45, 314 (1999).

[8] A. Malevanets and J. M. Yeomans, Faraday Discussions 112, 237 (1999).

[9] C. Denniston, E. Orlandini, and J. M. Yeomans, Europhys. Lett. 52, 481 (2000).

[10] E. Orlandini, M. R. Swift, and J. M. Yeomans, Europhys. Lett. 32, 463 (1995).

[11] M. R. Swift, E. Orlandini, W. R. Osborn, and J. M. Yeomans, Phys. Rev. E 54, 5041 (1996).

[12] V. M. Kendon, J. C. Desplat, P. Bladon, and M. E. Cates, Phys. Rev. Lett. 83, 576 (1999); V. M. Kendon, M. E. Cates, J. C. Desplat, I. Pagonabarraga, and P. Bladon, J. Fluid. Mech. 440, 147 (2001).

[13] H. Furukawa, Adv. Phys. 34, 703 (1985).

[14] A. J. Bray, Adv. Phys. 43, 357 (1994).

[15] Q. Zou, S. Hou, S. Chen, and G. D. Doolen, J. Stat. Phys. 81, 35 (1995).

[16] H. He, L. S. Luo and M. Dembo, Physica A 239, 276 (1997).

[17] H. He and L. S. Luo, J. Stat. Phys. 88, 927 (1997).

[18] H. Fang, R. Wan, and Z. Lin, Phys. Rev. E 66, 036314 (2002).

[19] P. Bhatnagar, E. P. Gross, and M. K. Krook, Phys. Rev. 94, 511 (1954).

[20] H. Chen, S. Chen, and W. Matthaeus, Phys. Rev. A 45, R5339 (1992).

[21] Aiguo Xu, G. Gonnella, and A. Lamura, Phys. Rev. E 67, 056105 (2003).

[22] S. Chapman and T. Cowling, The Mathematical Theory of Non-uniform Gases (Cambridge University Press, Cambridge, 1970).

[23] D. O. Martinez, W. H. Matthaeus, S. Chen, and D. C. Montgomery, Phys. Fluids 6, 1285 (1994).

[24] I. M. Lifshitz and V. V. Slyozov, J. Phys. Chem. Solids 19, 35 (1961).

[25] E. D. Siggia, Phys. Rev. A 20, 595 (1979).

[26] N. Gonzalez-Segredo, M. Nekovee, and P. V. Coveney, preprint, cond-mat/0301046, in press in Phys. Rev. E. 


\section{TABLES}

\begin{tabular}{cccccc}
\hline \hline set & $\Delta t$ & $-a, b$ & $\kappa$ & $\tau$ & $\Gamma$ \\
\hline 1 & 1 & 0.0625 & 0.04 & 2 & 1 \\
2 & 1 & 0.0625 & 0.04 & 2 & 0.2 \\
3 & 1 & 0.00625 & 0.004 & 0.5195 & 2.5 \\
4 & 0.2 & $1.252 \times 10^{-4}$ & $8 \times 10^{-5}$ & 0.521 & 40 \\
5 & 1 & 0.00313 & 0.002 & 0.542 & 8 \\
6 & 1 & 0.00125 & 0.0008 & 0.5015 & 10 \\
\hline \hline
\end{tabular}

TABLE I. The sets of parameter used in Figure 1. 


\begin{tabular}{ccccccc}
\hline \hline$\Delta t$ & $-a, b$ & $\kappa$ & $\tau$ & $\Gamma$ & $t_{\max }^{A}$ & $t_{\max }^{C}$ \\
\hline 1 & 0.083 & 0.053 & 4.73 & 0.1 & $>140460$ & $>146450$ \\
1 & 0.0625 & 0.04 & 2 & 0.2 & $=10139$ & $>184200$ \\
1 & 0.0625 & 0.04 & 1.1 & 0.3 & $=2043$ & $>10640$ \\
1 & 0.00625 & 0.004 & 0.575 & 4 & $=2983$ & $>6000$ \\
1 & 0.00625 & 0.004 & 0.5195 & 2.5 & $=148$ & $>61000$ \\
0.2 & $1.252 \times 10^{-4}$ & $8 \times 10^{-5}$ & 0.521 & 40 & $=41.6$ & $>1315$ \\
1 & 0.00313 & 0.002 & 0.542 & 8 & $=682$ & $>10200$ \\
1 & 0.00125 & 0.0008 & 0.5015 & 10 & $=97$ & $=2120$ \\
\hline \hline
\end{tabular}

TABLE II. Safe running times for the schemes A and C for 8 sets of parameters. $t_{\text {max }}$, where the upper label refers to the scheme used, is the longest simulated time. The symbol = means that the simulation becomes unstable after that time; the symbol $>$ means that the simulation is still stable up to that time. 


\section{FIGURES}
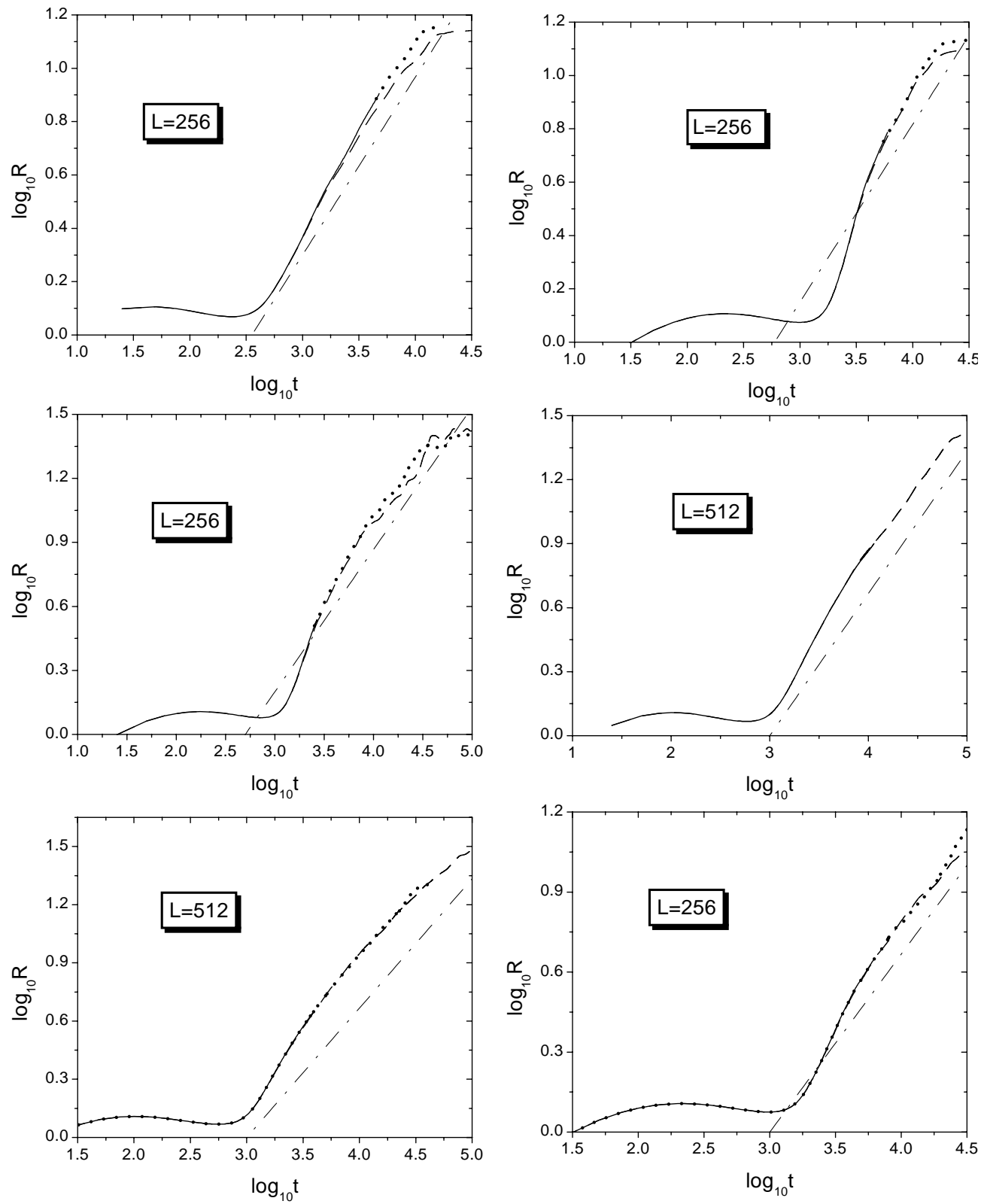

FIG. 1. $R(t)$ behavior for the sets of parameters listed in Table I. The solid, dashed, and dotted lines correspond to scheme A, B, and C, respectively. Runs with the schemes A and B always start from random initial configurations. The scheme $\mathrm{C}$ is applied either to simulations from random initial conditions (sets 5 and 6) or starting from a configuration slightly before the scheme A fails to work. Runs with the scheme $\mathrm{C}$ were eventually stopped, though still stable, once the system was phase separated. 
Fig.2 and Fig3 are in JPG format. Captions of Fig.2 and Fig. 3 are as follows,

Fig. 2 Configurations of the order parameter $\varphi(\mathrm{a})-(\mathrm{b})$, the total density $n$ (c)-(d), and velocity fields (e)-(f) for the parameter set 2 at the time $t=5895$. Figures (a), (c), (e) are for the scheme A, while (b), (d), (f) are for the scheme B. In picture (a) it is $-2.13 \leq \varphi \leq 1.77$ and in (b) it is $-1.06 \leq \varphi \leq 1.05$.

Fig. 3 Three-dimensional plot of the total density $n$ in a case with walls at time $t=1315$ using the scheme B. The parameters are those of set 4 in Table I. 
This figure "Fig2.jpg" is available in "jpg" format from: http://arxiv.org/ps/cond-mat/0309517v1 
This figure "Fig3.jpg" is available in "jpg" format from: http://arxiv.org/ps/cond-mat/0309517v1 